\title{
Submucosal squamous cell carcinoma of the oral tongue presenting as lingual abscess
}

\author{
Erin Mulry ${ }^{1}$, Solomon Husain ${ }^{2}$, Adam Gigliotti ${ }^{2}$, Kevin Leahy ${ }^{2}$, Rabie Shanti ${ }^{2,3}$ and Karthik Rajasekaran ${ }^{2 *}$ \\ ${ }^{1}$ University of Connecticut School of Medicine, 200 Academic Way, Farmington, CT 06032, USA \\ ${ }^{2}$ Department of Otorhinolaryngology-Head and Neck Surgery, University of Pennsylvania, 800 Walnut St, 18th floor, Philadelphia, PA 19104, USA \\ ${ }^{3}$ Department of Oral and Maxillofacial Surgery, University of Pennsylvania, 3400 Civic Center Boulevard South Pavilion, 4th floor, Philadelphia, PA 19104, USA
}

\begin{abstract}
Squamous cell carcinoma (SCC) of the tongue typically presents with mucosal lesions. Here, we describe a patient presenting with a tender mass of the tongue and intermittent fevers, though with normal overlying mucosa on exam. Computed tomography $(\mathrm{CT})$ revealed what appeared to be a lingual abscess, and she was brought to the operating room (OR) for incision and drainage (I\&D). Given the concerns for an occult cancer, a biopsy was performed and revealed SCC. Here we discuss the implications of this bizarre presentation of a tongue malignancy and review published literature on similar cases. Thus far, this is the first reported case of a primary tongue SCC lacking mucosal involvement.
\end{abstract}

\section{Introduction}

SCC of the oral tongue accounts for about half of oral cavity SCC [1]. Major risk factors include tobacco smoking and alcohol use [1]. Oral tongue SCC often presents with premalignant lesions such as leukoplakia or erythroplakia, seen as white or red mucosal changes to the tongue [2]. In this report, we describe a SCC of the oral tongue with complete absence of mucosal involvement, presenting initially as a lingual abscess.

\section{Case report}

A 62 y/o female with a 78 pack-year smoking history presented with 4 weeks of a painful mass of the right posterior tongue, with pain radiating to the right ear. She also noted intermittent fevers, though denied any growth in the mass, dysphagia, or dyspnoea. She had just completed 10 days of amoxicillin from an urgent care clinic with no improvement. Her medical history included rheumatoid arthritis, gastroesophageal reflux disease, and no history of head or neck surgeries. Exam was notable for tender fullness limited to the oral tongue, just right of midline, which was mobile and mildly fluctuant though without any mucosal changes (Figure 1). Labs were normal. CT of the neck revealed a $1.7 \mathrm{~cm}$ ring-enhancing lesion with surrounding oedema to right oral tongue with no lymphadenopathy (Figure 2 ). The lesion was identified as likely an abscess, though could not rule out neoplasm. The patient was then taken to the OR for I\&D of the tongue lesion with drainage of purulent fluid, during which biopsy and cultures were taken. Cultures returned as mouth flora, though biopsy revealed p16 positive invasive SCC. This patient's lack of mucosal changes on exam suggests this neoplasm is a submucosal oral tongue SCC.

Workup included a positron emission tomography (PET) scan, which showed FDG avid lesions to the right tongue and left perihilar lung with an adjacent confluent nodule, favoured to represent an independent primary bronchogenic malignancy with an adjacent hilar lymph node metastasis.
Since the tumour was located just right of midline, a near-total anterior glossectomy was planned in order to clear the tumour with negative margins. The tip of the tongue, its associated vasculature, and a branch of the hypoglossal nerve that innervated the tip was preserved. The defect was reconstructed with a radial forearm free flap (Figure 3). The patient also underwent bilateral selective neck dissections since the tumour felt to abut the midline intraoperatively. She was discharged home post-operative day 6 in good condition.

Final pathology revealed a pT3 N2b scc with a depth of invasion of $25 \mathrm{~mm}$ (Figure 4). Three nodes were positive for SCC without extranodal extension, and the primary tumour was positive for perineural and lymph-vascular invasion. The patient is scheduled to receive adjuvant chemoradiation therapy.

In order to conduct a literature review, articles published on PubMed were chosen based on relevance of their title and abstract. Articles found to discuss lingual abscesses, head and neck malignancies with infectious presentations, and unusual presentation of oral cavity SCCs were summarized and compared with the case we present here.

\section{Discussion and conclusion}

After an extensive search of published literature, this appears to be only the second report of SCC of the oral tongue that lacks mucosal involvement. It also appears to be the first reported case of a head and neck malignancy presenting as a lingual abscess.

${ }^{\star}$ Correspondence to: Karthik Rajasekaran, Department of OtorhinolaryngologyHead and Neck Surgery, University of Pennsylvania, Philadelphia, USA, Tel: 215-829-5180; E-mail: Karthik.Rajasekaran@pennmedicine.upenn.edu

Key words: head and neck squamous cell carcinoma, lingual abscess, oral cancer, submucosal squamous cell carcinoma, neoplasm presenting as abscess

Received: February 07, 2020; Accepted: February 21, 2020; Published: February 25,2020 


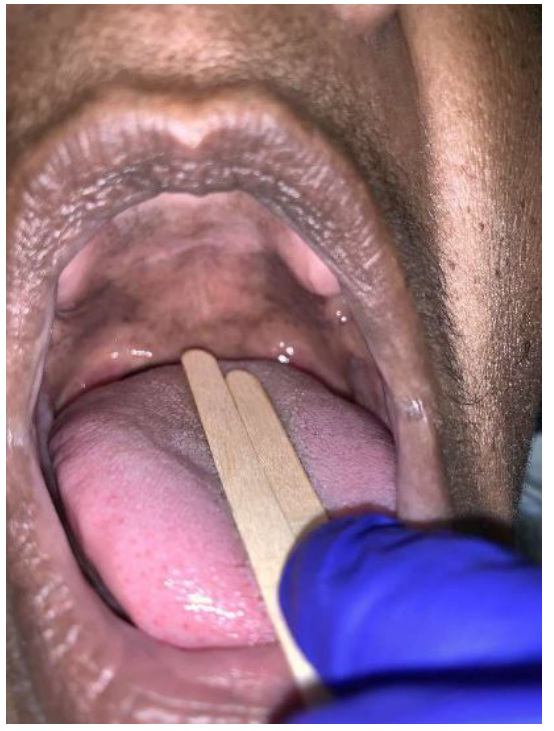

Figure 1. Inspection of the oral tongue reveals no mucosal changes.
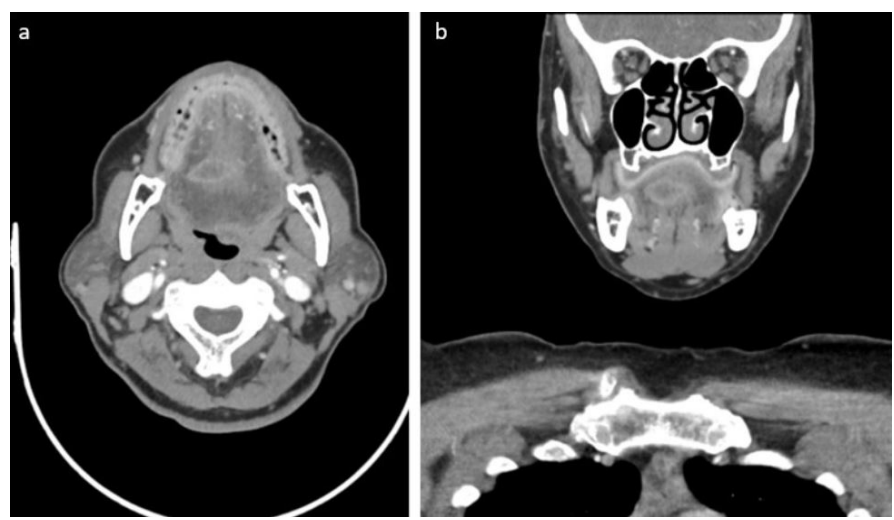

Figure 2. Axial (a) and coronal (b) $\mathrm{CT}$ images showing $1.7 \mathrm{~cm}$ ring-enhancing lesion with surrounding oedema to right oral tongue.

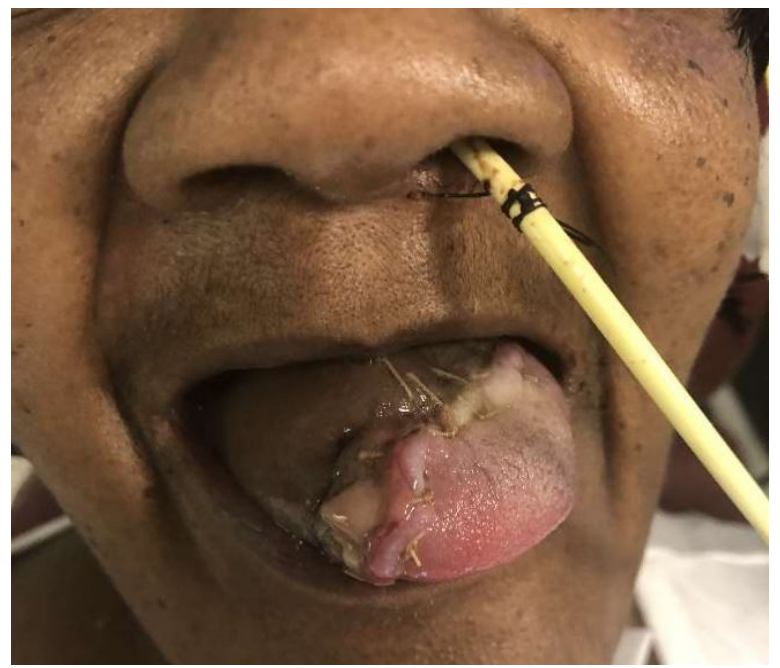

Figure 3. Post-operative anterior two-thirds glossectomy with tip preservation and radial forearm free flap reconstruction.

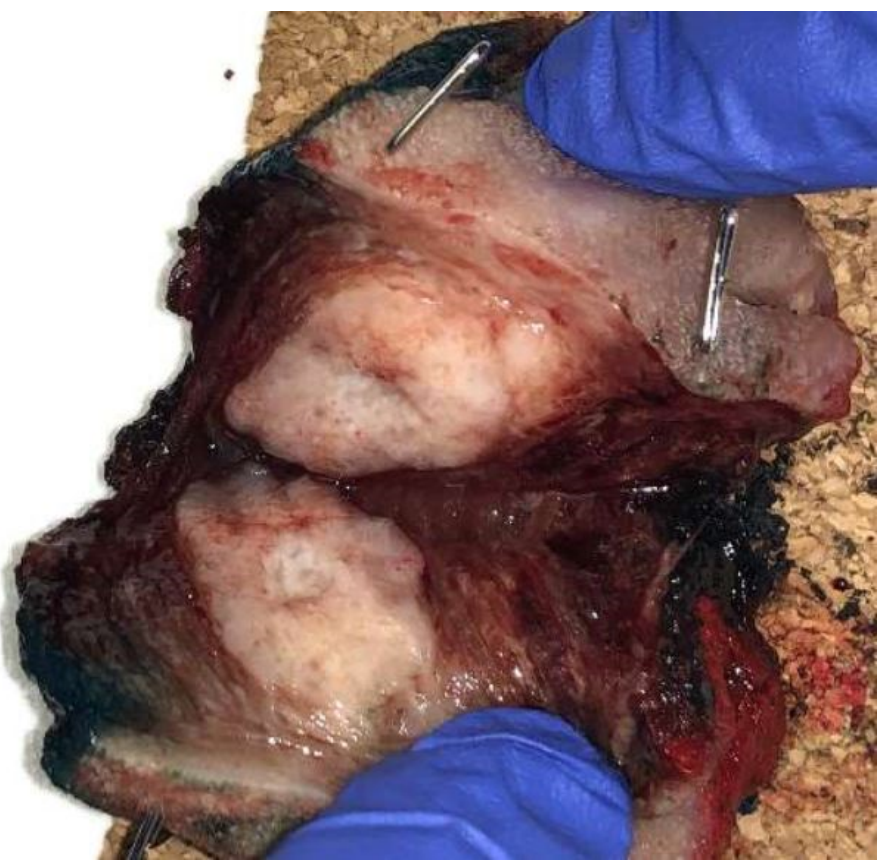

Figure 4. Tumour post resection, bisected showing no mucosal involvement.

Normally, abscesses of the tongue are associated with predisposing factors including local trauma, heavy smoking, and poor oral hygiene [3]. While uncommon, there have been reports of head and neck malignancies presenting with an abscess, though none located in the tongue. One study describes a patient with a parapharyngeal abscess which was culture positive for staph aureus, though 10 months later presented with a $5 \mathrm{~cm}$ neck mass under the I\&D scar and a large supraglottic tumour, both positive for SCC [4]. Another study did a retrospective review of 40 patients with deep neck abscesses, of which 5 were found to have an associated head and neck malignancy [5]. These reports as well as the case we present here highlight the importance of biopsy in the initial workup of head and neck abscesses.

The lack of mucosal involvement of this patient raises questions on the origin of this SCC, one possibility being that the SCC was a metastatic lesion. Metastasis of primary cancers to the oral cavity occurs only $1 \%$ of the time, most commonly with primary lung or breast cancer [6]. One study presents a patient presenting similarly with tongue pain and an extensive smoking history, found to have a submucosal SCC of the posterolateral tongue [6]. This patient was later found to have a large lung mass on chest X-ray, which was histologically identical to the tongue mass on biopsy [6]. The patient was also found to have distant metastatic lesions, leading to a diagnosis of primary lung SCC with advanced metastatic disease [6]. While the patient we describe here did have an FDG avid lung lesion on PET scan, biopsy revealed a bronchogenic cancer rather than a metastatic lesion from the tongue.

This case report highlights the importance of maintaining a high suspicion of malignancy when working up unusual presentations of head and neck conditions such as a lingual abscess. With discussion of this unusual infectious presentation as well as identification of an oral tongue SCC with a complete absence of mucosal involvement, we aim to create awareness of this extremely rare presentation of oral cavity malignancy in order to improve future diagnosis and management. 


\section{References}

1. Ashraf MJ, Hosseini S, Monabati A, Valibeigi B, Khademi B, et al. (2017) The prevalence of human papilloma virus in squamous cell carcinoma of oral tongue. Iran J Pathol 2017;12: 144-149. [Crossref]

2. Thomson PJ, Goodson ML, Smith DR (2017) Profiling cancer risk in oral potentially malignant disorders - a patient cohort study. J Oral Path Med 46: 888-895. [Crossref]

3. Srivanitchapoom C, Yata K (2017) Lingual abscess: predisposing factors, pathophysiology, clinical manifestations, diagnosis, and management. Int $J$ Otolaryngol: 4504270
4. Lee WC, Walsh RM, Tse A (1996) Squamous cell carcinoma of the pharynx and larynx presenting as a neck abscess or cellulitis. J Laryngol Otology 100: 893-895. [Crossref]

5. Ridder GJ, Eglinger CF, Sander A, Technau-Ihling K (200) Neck abscess as primary manifestation of head and neck carcinoma: implications for diagnostic management Laryngorhinootologie 79: 604-608. [Crossref]

6. Terashima T, Matsuzaki T, Ichiro K, Nishida J, Tanaka Y, et al. (2004) Tongue metastasis as an initial presentation of a lung cancer. Inter Med 43: 727-730. [Crossref]

Copyright: (C2020 Mulry E. This is an open-access article distributed under the terms of the Creative Commons Attribution License, which permits unrestricted use, distribution, and reproduction in any medium, provided the original author and source are credited. 